\title{
ON FOUR INEQUALITIES IN SYMMETRIC FUNCTIONS
}

\author{
by J. B. McLEOD \\ (Received 16th March 1959)
}

1. LET us denote by $\alpha$ the set of $n$ real numbers $a_{1}, \ldots, a_{n}$, and by $c_{k}(\alpha)$ and $h_{k}(\alpha)$ the elementary and complete symmetric functions of degree $k$ in $\alpha_{1}, \ldots, a_{n}$, and by $c_{k}(\alpha)$ and $h_{k}(a)$ the elementary and complete symmetric functions of degree $k$ in $a_{1}, \ldots, a_{n}$, i.e. $c_{k}(\alpha)$ is the sum of all possible products of $k$ different $a_{i}$ and $h_{k}(\alpha)$ is the sum of all possible products of $k a_{i}$, where now in any product one or more $\alpha_{i}$ may be repeated any number of times. We shall prove, for $\alpha_{i} \geqslant 0, \beta_{i} \geqslant 0$, that the following inequalities hold :

$$
\begin{aligned}
& \text { (i) }\left\{c_{k}(\boldsymbol{\alpha})\right\}^{\frac{1}{\bar{k}}}+\left\{c_{k}(\boldsymbol{\beta})\right\}^{\frac{1}{k}} \leqslant\left\{c_{k}(\boldsymbol{\alpha}+\boldsymbol{\beta})\right\}^{\frac{1}{k}} \\
& \text { (ii) }\left\{\frac{c_{k+l}(\boldsymbol{\alpha})}{c_{l}(\boldsymbol{\alpha})}\right\}^{\frac{1}{k}}+\left\{\frac{c_{k+l}(\boldsymbol{\beta})}{c_{l}(\boldsymbol{\beta})}\right\}^{\frac{1}{k}} \leqslant\left\{\frac{c_{k+l}(\boldsymbol{\alpha}+\boldsymbol{\beta})}{c_{l}(\boldsymbol{\alpha}+\boldsymbol{\beta})}\right\}^{\frac{1}{k}} \\
& \text { (iii) }\left\{h_{k}(\boldsymbol{\alpha})\right\}^{\frac{1}{\bar{k}}}+\left\{h_{k}(\boldsymbol{\beta})\right\}^{\frac{1}{k}} \geqslant\left\{h_{k}(\boldsymbol{\alpha}+\boldsymbol{\beta})\right\}^{\frac{1}{\bar{k}}}
\end{aligned}
$$

The first inequality is, of course, a particular case of the second, but it will make for a more intelligible exposition if we prove the first in detail and then use the ideas so developed to prove the others in succession.

To the best of my knowledge, no proof of the second or third inequalities exists so far in the literature, but the first has been recently proved in (1) by a different method from that which we shall adopt. It is also conjectured that there is a fourth inequality which extends (iii) in the same way as (ii) extends (i), namely

$$
\left\{\frac{h_{k+l}(\boldsymbol{\alpha})}{h_{l}(\boldsymbol{\alpha})}\right\}^{\frac{1}{k}}+\left\{\frac{h_{k+l}(\boldsymbol{\beta})}{h_{l}(\boldsymbol{\beta})}\right\}^{\frac{1}{k}} \geqslant\left\{\frac{h_{k+l}(\boldsymbol{\alpha}+\boldsymbol{\beta})}{h_{l}(\boldsymbol{\alpha}+\boldsymbol{\beta})}\right\}^{\frac{1}{k}},
$$

but the truth of this remains unproved.

I am much indebted to Professor A. C. Aitken, not only for suggesting the topic of these inequalities, but also for directing my attention to the work of Sylvester and Boole on unisignant determinants, on which the proofs given below hinge.

2. Let us first introduce the following notation. Let $c_{k-1 ; 1}(\alpha)$ [or, more shortly, just $c_{k-1 ; 1}$ ] denote the elementary symmetric function of degree $k-1$ in $a_{2}, a_{3}, \ldots, a_{n}$; and similarly for $c_{k-1 ; r}(\alpha)$. Let $c_{k-2 ; 1,2}(\alpha)$ [or, more shortly, just $c_{k-1 ; 1,2}$ ] denote the elementary symmetric function of degree $k-2$ in $a_{3}, a_{4}, \ldots, a_{n}$; and similarly for $c_{k-2 ; r, 8}(\alpha)$. If $r=s$, we define $c_{k-2 ; r, s}$ to be zero.

We shall prove the following

Lemma. The determinant $\left|c_{k-2 ; r, 8}(\alpha)\right|$ is unisignant, each term in the E.M.S. - 0 
expansion having the sign $(-1)^{n-1}$. (By saying that a determinant is unisignant, we mean that on expansion it consists of a sum of terms each of the same sign, this sign being either positive or negative according to the context.)

Proof. An equivalent statement is that the determinant $\left|-c_{k-2 ; r, s}\right|$ is negatively unisignant. If we denote $\sum_{s=1}^{n} c_{k \div 2 ; r, s}$ by $\Sigma_{r}$, then we may write $\left|-c_{k-2 ; r, s}\right|$ as

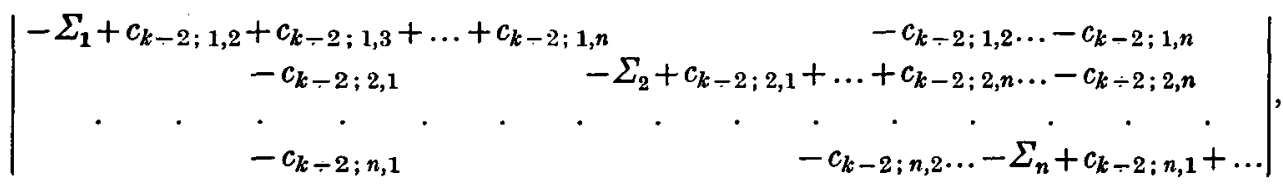

when it takes a form discussed by Sylvester (2). [See also Muir, Theory of Determinants, Vol. II, p. 457 et seq.] Sylvester gives a rule for evaluating such a determinant which is as follows : the value of the determinant is given by formally multiplying together the elements on the diagonal and rejecting in the expansion of this product all terms containing a cycle of indices, i.e. any term involving

$$
c_{k-2 ; r, 8} c_{k-2 ; 8, r} \text { or } c_{k-2 ; r, 8} c_{k-2 ; 8, t} c_{k-2 ; t, r}
$$

would be rejected, and similarly for higher cycles.

In our application of Sylvester's rule, we notice that the diagonal elements (and hence their product) are equal to zero, and that the value of the determinant is therefore given by the negative of the terms rejected by the rule. To prove the lemma, we must show that such rejected terms as do not cancel one another are positively unisignant. is

Let us look at the product of the diagonal terms more closely. The product

$$
\Pi_{r}\left(-\Sigma_{r}+c_{k-2 ; r, 1}+c_{k-2 ; r, 2}+\ldots+c_{k-2 ; r, n)} .\right.
$$

If we choose $\Sigma_{r}$ from each bracket, then no cycles of indices can occur, there are therefore no rejections, and we need not consider this further. This applies again if we choose $\Sigma_{r}$ from every bracket except one.

To consider what happens when we choose $\Sigma_{r}$ from fewer brackets, i.e. from $n-2$ or less, let us denote the aggregate of all terms rejected when we choose $\Sigma_{r}$ from every bracket except $m$ by $S_{m}$. Thus $S_{m}$ consists of the sum of all terms which may be obtained by choosing $\Sigma_{r}$ from every bracket except $m$ and which contain one or more cycles of indices.

Then in $S_{2}$ the only type of cycle possible is a cycle on two indices, and so a typical term in $S_{3}$ is

$$
(-1)^{n-2} \Sigma_{1} \Sigma_{2} \ldots \Sigma_{n-2}\left(c_{k-2 ; n-1, n} c_{k-2 ; n, n-1}\right) \text {. }
$$

In $S_{3}$, cycles on three indices are possible, but also, of course, cycles on two indices, and amongst the latter is a typical term

$$
(-1)^{n-3} \Sigma_{1} \Sigma_{2} \ldots \Sigma_{n-3} c_{k-2 ; n-2, r}\left(c_{k-2 ; n-1, n} c_{k-2 ; n, n-1}\right)
$$


for any $r$. Adding these terms for all possible values of $r$, we obtain

$$
(-1)^{n-3} \Sigma_{1} \Sigma_{2} \ldots \Sigma_{n-3} \Sigma_{n-2}\left(c_{k-2 ; n-1, n} c_{k-2 ; n, n-1}\right) \text {, }
$$

which cancels the typical term above in $S_{2}$. Thus the terms in $S_{3}$ more than cancel those in $S_{2}$, and the sum $S_{3}+S_{2}$ is unisignant with sign that of $S_{3}$, i.e. $(-1)^{n-3}$.

More generally, every type of cycle or combination of cycles possible in $S_{m-1}$ is possible also in $S_{m}$, along with new combinations, and, by the same argument as in the last paragraph, the appearance of any cycle or combination of cycles in $S_{m-1}$ is cancelled by its appearance in $S_{m}$, so that $S_{m}+S_{m-1}$ is unisignant with sign that of $S_{m}$, i.e. $(-1)^{n-m}$.

Thus the terms rejected by Sylvester's rule are in all

$$
S_{n}+S_{n-1}+\ldots+S_{2}
$$

and, if we pair these as

$$
\left(S_{n}+S_{n-1}\right)+\left(S_{n-2}+S_{n-3}\right)+\ldots
$$

we see from the above paragraph that the rejected terms are positively unisignant, which proves the lemma.

3. As a further preliminary to the proof of the first inequality, we observe that we may assume throughout the proof that the inequality is satisfied if the vectors $\alpha, \beta$ are of dimension less than $n$. For, if we succeed in using this as an induction hypothesis to prove that the inequality also holds when $\alpha, \boldsymbol{\beta}$ are of dimension $n$, then we know that the inequality must hold whatever the dimension of $\alpha, \beta$ provided that it holds when their dimension is one. But this last is trivial, for either $k=1$ and both sides of the inequality become $a_{1}+\beta_{1}$, or $k>1$ and both sides of the inequality become zero. The induction hypothesis that the inequality holds when $\alpha, \beta$ are of dimension less than $n$ will therefore be assumed without further mention in what follows.

We shall now use this induction hypothesis to prove first that the inequality holds for $\alpha, \beta$ of dimension $n$ when one or more $\alpha_{i}$ and one or more $\beta_{i}$ vanish simultaneously. The corresponding results for the other two inequalities will also be required later, and it will be convenient to prove all three results in this section.

In the case of the first inequality, we may suppose without loss of generality that $a_{1}=0$. If $\beta_{1}=0$, the result follows at once from the induction hypothesis, since in effect both $\alpha, \beta$ reduce to dimension $n-1$. If $\beta_{1} \neq 0$, we may suppose without loss of generality that $\beta_{2}=0$.

Now introduce the vectors $\alpha^{*}, \beta^{*}$, where $\alpha^{*}=\alpha$, and $\beta^{*}$ is the same as $\beta$ except that the first and second coordinates are interchanged. Then we have

$$
c_{k}\left(\alpha^{*}\right)=c_{k}(\boldsymbol{\alpha}), \quad c_{k}\left(\boldsymbol{\beta}^{*}\right)=c_{k}(\boldsymbol{\beta}),
$$

and, since $\alpha^{*}, \beta^{*}$ have $\alpha_{1}^{*}=\beta_{1}^{*}=0$ and so are in effect of dimension $n-1$, we obtain, using the induction hypothesis, that

$$
\left\{c_{k}(\alpha)\right\}^{\frac{1}{\bar{k}}}+\left\{c_{k}(\boldsymbol{\beta})\right\}^{\frac{1}{k}}=\left\{c_{k}\left(\boldsymbol{\alpha}^{*}\right)\right\}^{\frac{1}{\bar{k}}}+\left\{c_{k}\left(\boldsymbol{\beta}^{*}\right)\right\}^{\frac{1}{\bar{k}}} \leqslant\left\{c_{k}\left(\boldsymbol{\alpha}^{*}+\boldsymbol{\beta}^{*}\right)\right\}^{\frac{1}{k}}
$$


The result we require is therefore proved if we can show that

i.e. that

$$
\left\{c_{k}\left(\boldsymbol{a}^{*}+\boldsymbol{\beta}^{*}\right)\right\}^{\frac{1}{k}} \leqslant\left\{c_{k}(\boldsymbol{\alpha}+\boldsymbol{\beta})\right\}^{\frac{1}{k}}
$$

$$
c_{k}\left(\boldsymbol{\alpha}^{*}+\boldsymbol{\beta}^{*}\right) \leqslant c_{k}(\boldsymbol{\alpha}+\boldsymbol{\beta}) \text {. }
$$

But this last inequality is certainly true, for the two sides contain the same terms except that there are terms on the right-hand side involving the product $\alpha_{2} \beta_{1}$, and such terms cannot occur on the left-hand side.

This scheme of argument applies equally well for the other two inequalities, with only the final step in the proof requiring separate consideration. In the case of the second inequality, we have to show that

i.e. that

$$
\frac{c_{k+l}\left(\alpha^{*}+\beta^{*}\right)}{c_{l}\left(\alpha^{*}+\beta^{*}\right)} \leqslant \frac{c_{k+l}(\alpha+\beta)}{c_{l}(\alpha+\beta)}
$$

$$
c_{l}(\boldsymbol{\alpha}+\boldsymbol{\beta}) c_{k+l}\left(\boldsymbol{\alpha}^{*}+\boldsymbol{\beta}^{*}\right) \leqslant c_{l}\left(\boldsymbol{\alpha}^{*}+\boldsymbol{\beta}^{*}\right) c_{k+l}(\boldsymbol{\alpha}+\boldsymbol{\beta}) .
$$

We have already seen that $c_{l}(\alpha+\beta)$ and $c_{l}\left(\alpha^{*}+\beta^{*}\right)$ differ only in terms involving $\alpha_{2} \beta_{1}$, so that we may write

$$
c_{l}\left(\boldsymbol{\alpha}^{*}+\boldsymbol{\beta}^{*}\right)=c_{l}(\boldsymbol{\alpha}+\boldsymbol{\beta})-a_{2} \beta_{1} c_{l-2 ; 1,2}(\boldsymbol{\alpha}+\boldsymbol{\beta}),
$$

and similarly

$$
c_{k+l}\left(\boldsymbol{\alpha}^{*}+\boldsymbol{\beta}^{*}\right)=c_{k+l}(\boldsymbol{\alpha}+\boldsymbol{\beta})-\alpha_{2} \beta_{1} c_{k+l-2 ; 1,2}(\boldsymbol{\alpha}+\boldsymbol{\beta}) .
$$

Then the inequality required becomes

$$
c_{l}(\boldsymbol{\alpha}+\boldsymbol{\beta}) c_{k+l-2 ; 1,2}(\boldsymbol{\alpha}+\boldsymbol{\beta})-c_{k+l}(\boldsymbol{\alpha}+\boldsymbol{\beta}) c_{l-2 ; 1,2}(\boldsymbol{\alpha}+\boldsymbol{\beta}) \geqslant 0,
$$

where the argument $\alpha+\beta$ may now safely be omitted. The terms involving $\alpha_{2} \beta_{1}$ in the left-hand side are

$$
a_{2} \beta_{1}\left\{c_{l-2 ; 1,2} c_{k+l-2 ; 1,2}-c_{k+l-2 ; 1,2} c_{l-2 ; 1,2}\right\},
$$

which is obviously zero; the terms involving $\alpha_{2}$ (but not $\beta_{1}$ ) are

$$
a_{2}\left\{c_{l-1 ; 1,2} c_{k+l-2 ; 1,2}-c_{k+l-1 ; 1,2} c_{l-2 ; 1,2}\right\} ;
$$

those involving $\beta_{1}$ (but not $a_{2}$ ) are

$$
\beta_{1}\left\{c_{l-1 ; 1,2} c_{k+l-2 ; 1,2}-c_{k+l-1 ; 1,2} c_{l-2 ; 1,2}\right\}
$$

those involving neither $\alpha_{2}$ nor $\beta_{1}$ are

$$
c_{l ; 1,2} c_{k+l-2 ; 1,2}-c_{k+l ; 1,2} c_{l-2 ; 1,2}
$$

and that each of (3.1), (3.2) and (3.3) are non-negative follows at once from the lemma which closes this section.

In the case of the third inequality, the inequality to be proved is that

$$
h_{k}\left(\boldsymbol{\alpha}^{*}+\boldsymbol{\beta}^{*}\right) \geqslant h_{k}(\boldsymbol{\alpha}+\boldsymbol{\beta}) .
$$

Now on the left-hand side those terms involving $a_{2}, \beta_{1}$ to a total degree of $m$ $(0 \leqslant m \leqslant k)$ are just those terms involving $\left(\alpha_{2}+\beta_{1}\right)^{m}$. On the right-hand side, in the corresponding terms, $\left(\alpha_{2}+\beta_{1}\right)^{m}$ is replaced by

$$
a_{2}{ }^{m}+a_{2}{ }^{m-1} \beta_{1}+\ldots+\beta_{1}{ }^{m} \text {. }
$$




\section{ON FOUR INEQUALITIES IN SYMMETRIC FUNCTIONS}

Since

$$
\left(\alpha_{2}+\beta_{1}\right)^{m} \geqslant \alpha_{2}^{m}+\alpha_{2}^{m-1} \beta_{1}+\ldots+\beta_{1}^{m}
$$

the inequality is proved.

Lemma. If $p+q=r+s(p \geqslant q, r \geqslant s)$, then $c_{p} c_{q}-c_{r} c_{s}$ is positively unisignant if $p-q<r-s$, whatever may be the dimension of the vector which is the argument of $c_{p}, c_{q}, c_{r}, c_{s}$.

Proof. The proof is by induction. We suppose the lemma true when $p+q \leqslant m$, and then prove it for $p+q=m+1$. It is easily seen to be true in the first applicable case, i.e. when $p+q=r+s=2$ and $p=2, q=0, r=s=1$.

Suppose then that $p+q=m+1$. We will consider the coefficients of $a_{j}^{2}, a_{j}$ in $c_{p} c_{q}-c_{r} c_{s}$, for any $j$. If they are positive, then the result clearly follows.

The coefficient of $\alpha_{j}^{2}$ is

$$
c_{p-1 ; j} c_{q-1 ; j}-c_{r-1 ; j} c_{8-1 ; j},
$$

which is positive by the induction hypothesis, and the same type of argument shows that the coefficient of $\alpha_{j}$ is positive.

4. To prove the first inequality of $\S 1$, we consider $\alpha+\beta$ to be fixed. We set $\boldsymbol{\alpha}+\boldsymbol{\beta}=\boldsymbol{a}=\left(a_{1}, \ldots, a_{n}\right)$, and we shall assume that $a_{i} \neq 0(i=1, \ldots, n)$. (This is no restriction, for if we prove the inequality in this case, then from continuity the inequality still holds when one or more $a_{i}$ is zero.) With $\alpha+\beta=a$ thus fixed, we may regard $\beta=a-\alpha$ as a function of $\alpha$, and we have to prove that in the region $R$ defined by $0 \leqslant a_{i} \leqslant a_{i}(i=1, \ldots, n)$

$$
\left.\left\{c_{k}(\alpha)\right\}^{\frac{1}{\vec{k}}}+\left\{c_{k}(a-\alpha)\right\}^{\frac{1}{\vec{k}}} \leqslant c_{k}(a)\right\}^{\frac{1}{\vec{k}}}
$$

The left-hand member is a continuous function of $\alpha$, and two possibilities arise. The first is that it attains its greatest value on the boundary of $R$, and we shall prove in $\$ 5$ that the inequality is satisfied on this boundary.

Alternatively, any point $\alpha$ at which the left-hand member above attains its greatest value must lie strictly inside $R$, and so must be a stationary value of the function. Our next task will be to prove that inside $R$ the only stationary points occur when $\alpha, \beta$ are proportional, when, as is at once obvious, the inequality becomes equality. This will complete the proof of the inequality.

The usual necessary conditions for a stationary point are here

$$
\frac{1}{k} \frac{c_{k-1 ; i}(\alpha)}{\left\{c_{k}(\alpha)\right\}^{1-\frac{1}{k}}}-\frac{1}{k} \frac{c_{k-1 ; i}(a-\alpha)}{\left\{c_{k}(a-\alpha)\right\}^{1-\frac{1}{k}}}=0(i=1, \ldots, n) .
$$

Since this set of equations is dimensionless, we may set $a-\alpha=K \gamma$, where $K$ is a constant to be suitably chosen, and we still have

$$
\frac{1}{k} \frac{c_{k-1 ; i}(\alpha)}{\left\{c_{k}(\alpha)\right\}^{1-\frac{1}{k}}}-\frac{1}{k} \frac{c_{k-1 ; i}(\gamma)}{\left\{c_{k}(\gamma)\right\}^{1-\frac{1}{k}}}=0
$$


If we now choose $K$ so that

$$
c_{k}(\alpha)=c_{k}(\gamma)
$$

then the set of equations simplifies to

$$
c_{k-1 ; i}(\alpha)=c_{k-1 ; i}(\gamma),
$$

and all that remains is to prove that the only solution of this set of equations is $\alpha=\gamma$. This will in fact be so if the Jacobian of the $n$ functions $c_{k \div 1 ; i}(\alpha)$ with respect to the $n$ variables $a_{i}$ does not vanish inside $R$. But this Jacobian is just the determinant of the lemma of $\S 2$.

5. We have now shown that if the inequality fails to hold at all, then it fails to hold on the boundary of $R$, i.e. when at least one $\alpha_{i}$ or $\beta_{i}$ is zero. In particular, we may suppose without loss of generality that at least one $\alpha_{i}$ is zero.

We shall now prove that if the inequality fails to hold when $p$ of the $a_{i}$ are zero, then it fails to hold when $p+1$ are zero. For, if we prove this, it will follow that if the inequality fails to hold at all, then it fails to hold when sufficient $\alpha_{i}$ are zero that $c_{k}(\alpha)=0$. But the inequality certainly does not fail then, and so it always holds.

Suppose then that $a_{1}, \ldots, a_{p}$ are zero. We shall consider first the surface $S$ in $R$ given by $a_{1}=\epsilon_{1}, \ldots, a_{p}=\epsilon_{p}$, where the $\epsilon_{i}$ are small but strictly positive constants. If the inequality fails to hold on this surface, then two possibilities arise. The first is that it fails to hold on the boundary of $S$, i.e. when at least one $\alpha_{i}$ or $\beta_{i}(i \neq 1, \ldots, p)$ is zero, and this, as we shall see later, is what we want.

The second possibility is that the inequality fails to hold strictly inside $S$, in which case the function

$$
\left\{c_{k}(\alpha)\right\}^{\frac{1}{k}}+\left\{c_{k}(a-\alpha)\right\}^{\frac{1}{k}}
$$

must attain its greatest value in $S$ at a stationary value strictly inside $S$. The necessary conditions for a stationary value are

$$
\frac{1}{k} \frac{c_{k-1 ; i}(\alpha)}{\left\{c_{k}(\alpha)\right\}^{1-\frac{1}{k}}}-\frac{1}{k} \frac{c_{k-1 ; i}(a-\alpha)}{\left\{c_{k}(a-\alpha)\right\}^{1-\frac{1}{k}}}=0 \quad(i=p+1, \ldots, n),
$$

and if again we put $a-\alpha=K \gamma$, so that $c_{k}(\alpha)=c_{k}(\gamma)$, we have

$$
c_{k-1 ; i}(\alpha)=c_{k-1 ; i}(\gamma) \text {. }
$$

Again, this has a unique solution, for the Jacobian of the $n-p$ functions $c_{k-1 ; i}(\alpha)$ with respect to the $n-p$ variables $a_{p+1}, \ldots, \alpha_{n}$ is a coaxial minor of the determinant of the lemma of $\$ 2$, and can be proved unisignant in the same way as the determinant itself was. We should of course like the unique solution of (5.1) to be $\alpha=\gamma$, but (5.1) in effect determines only the relationship between $a_{p+1}, \ldots, a_{n}$ and $\gamma_{p+1}, \ldots, \gamma_{n}$, and not the relationship between $a_{1}, \ldots, a_{p}$ and $\gamma_{1}, \ldots, \gamma_{p}$, which is determined by the choice of $\epsilon_{1}, \ldots, \epsilon_{p}$, and for a general choice of $\epsilon_{1}, \ldots, \epsilon_{p}$, this relationship between $\alpha_{1}, \ldots, \alpha_{p}$ and $\gamma_{1}, \ldots, \gamma_{p}$ may not be consistent with $\alpha=\gamma$. In fact, if $\alpha=\gamma$, then $\alpha$ is proportional to $a$, and since $a_{1}=\epsilon_{1}, \ldots, a_{p}=\epsilon_{p}$, it follows that $\epsilon_{1} / a_{1}=\epsilon_{2} / a_{2}=\ldots=\epsilon_{p} / a_{p}$, which may not be so. 
However, we are going to allow $\epsilon_{1}, \ldots, \epsilon_{p}$ to tend to zero, and it will not matter how they tend to zero. Let us therefore choose $\epsilon_{1}, \ldots, \epsilon_{p}$ so that $\epsilon_{1} / a_{1}=\ldots=\epsilon_{p} / a_{p}$, and then $\alpha=\gamma$ satisfies both this choice of $\epsilon_{1}, \ldots, \epsilon_{p}$ and the equations (5.1); i.e. $\alpha=\gamma$ is the unique solution of (5.1), and for this solution the inequality becomes equality.

Thus, with this choice of $\epsilon_{1}, \ldots, \epsilon_{p}$, we conclude that if the inequality fails to hold on $S$, then it fails to hold on the boundary of $S$. Allowing the $\epsilon_{i}$ to tend to zero, we have that if the inequality fails to hold when $p$ of the $a_{i}$ are zero, then either it fails to hold when $p+1$ or more are zero (which is what we want to prove) or it fails to hold when $p$ of the $\alpha_{i}$ are zero and one or more $\beta_{i}$ are zero, and this last possibility is excluded by $\S 3$. This completes the proof of the inequality.

\section{The Second Inequality}

It is our aim now to prove that, for $\alpha_{i} \geqslant 0, \beta_{i} \geqslant 0$,

$$
\left\{\frac{c_{k+l}(\boldsymbol{\alpha})}{c_{l}(\boldsymbol{\alpha})}\right\}^{\frac{1}{\bar{k}}}+\left\{\frac{c_{k+l}(\boldsymbol{\beta})}{c_{l}(\boldsymbol{\beta})}\right\}^{\frac{1}{k}} \leqslant\left\{\frac{c_{k+l}(\boldsymbol{\alpha}+\boldsymbol{\beta})}{c_{l}(\boldsymbol{\alpha}+\boldsymbol{\beta})}\right\}^{\frac{1}{k}}
$$

The scheme of the proof for this inequality is the same as that for the first inequality, and the two proofs differ only in the mechanical details. In fact, even these differ essentially only at two points; the first of these was dealt with in $\S 3$, and the second occurs when we show that, if we define the region $R$ as before, then

$$
\left\{\frac{c_{k+l}(\boldsymbol{\alpha})}{c_{l}(\boldsymbol{\alpha})}\right\}^{\frac{1}{k}}+\left\{\frac{c_{k+l}(\boldsymbol{a}-\boldsymbol{\alpha})}{c_{l}(\boldsymbol{a}-\boldsymbol{\alpha})}\right\}^{\frac{1}{\bar{k}}}
$$

regarded as a function of $\alpha$ for fixed $a$, cannot have a stationary value strictly inside $R$ other than one for which $\alpha, \beta, a$ are proportional.

The necessary conditions for $(6.1)$ to have a stationary value are

$$
\begin{aligned}
\frac{1}{k}\left\{\frac{c_{k+l}(\alpha)}{c_{l}(\alpha)}\right\}^{\frac{1}{k}-1}\left\{\frac{c_{k+l-1 ; i}(\alpha) c_{l}(\alpha)-c_{k+l}(\alpha) c_{l-1 ; i}(\alpha)}{\left[c_{l}(\alpha)\right]^{2}}\right\} \\
=\frac{1}{k}\left\{\frac{c_{k+l}(a-\alpha)}{c_{l}(a-\alpha)}\right\}^{\frac{1}{k}-1}\left\{\frac{c_{k+l-1 ; i}(a-\alpha) c_{l}(a-\alpha)-c_{k+l}(a-\alpha) c_{l-1 ; i}(a-\alpha)}{\left[c_{l}(a-\alpha)\right]^{2}}\right\} \\
(i=1, \ldots, n) .
\end{aligned}
$$

These equations are dimensionless, and so continue to hold if we replace $a-\alpha$ by $\gamma$, where $\gamma=K(a-\alpha)$, and $K$ is a constant chosen so that

$$
\left\{\frac{c_{k+l}(\alpha)}{c_{l}(\alpha)}\right\}^{\frac{1}{k}-1} \div\left[c_{l}(\alpha)\right]^{2}=\left\{\frac{c_{k+l}(\gamma)}{c_{l}(\gamma)}\right\}^{\frac{1}{\bar{k}}-1} \div\left[c_{l}(\gamma)\right]^{2}
$$

The equations then simplify to

where

$$
f_{i}(\alpha)=f_{i}(\gamma)
$$

$$
f_{i} \equiv c_{k+l-1 ; i} c_{l}-c_{k+l} c_{l-1 ; i}
$$


and all that remains to be proved is that the only solution to this set of equations is $\alpha=\gamma$. This will be so if the Jacobian of the $n$ functions $f_{i}(\alpha)$ with respect to the $n$ variables $\alpha_{i}$ does not vanish.

To examine this Jacobian more closely, we observe that

$$
\begin{aligned}
c_{l} & =\alpha_{i} c_{l-1 ; i}+c_{l ; i}, \\
c_{k+l} & =\alpha_{i} c_{k+l-1 ; i}+c_{k+l ; i},
\end{aligned}
$$

and substitute these in the definition of $f_{i}$ to obtain

$$
f_{i}=c_{k+l-1 ; i} c_{l ; i}-c_{k+l ; i} c_{l-1 ; i} \text {. }
$$

It is now clear that $f_{i}$ is independent of $\alpha_{i}$, and so the elements on the diagonal of the Jacobian are zero.

Furthermore, by the lemma at the end of $\S 3, f_{i}$, when expanded in a sum of terms, is positively unisignant, and hence $\partial f_{i} / \partial a_{i}(i \neq j)$ is positively unisignant. Now, in proving the unisignancy of the determinant $\left|c_{k-2 ; r, 8}\right|$ in $\$ 2$, the only two properties of the $c_{k-2 ; r, 8}$ which we used were that $c_{k-2 ; r, r}=0$ and that $c_{k-2 ; r, 8}(r \neq s)$ is strictly positive inside $R$. This is seen by repeating the proof with $c_{k-2 ; r, 8}$ replaced by $a_{r, 8}$, where the $a_{r, 8}$ are any numbers satisfying only the conditions $a_{r, r}=0$ and $a_{r, 8}(r \neq s)$ strictly positive inside $R$, and it will be observed that the proof continues to hold good. In particular, it continues to hold good for the determinant $\left|\partial f_{r} / \partial \alpha_{s}\right|$, which is accordingly unisignant, and the proof of the second inequality is complete.

\section{The Third Inequality}

It is now our aim to prove that

$$
\left\{h_{k}(\boldsymbol{\alpha})\right\}^{\frac{1}{\vec{k}}}+\left\{h_{k}(\boldsymbol{\beta})\right\}^{\frac{1}{k}} \geqslant\left\{h_{k}(\boldsymbol{\alpha}+\boldsymbol{\beta})\right\}^{\frac{1}{\vec{k}}} .
$$

As with the second inequality, we may restrict ourselves to showing that the function

$$
\left\{h_{k}(\boldsymbol{\alpha})^{\frac{1}{\bar{k}}}+\left\{h_{k}(\boldsymbol{a}-\boldsymbol{\alpha})\right\}^{\frac{1}{k}}\right.
$$

regarded as a function of $\alpha$ for fixed $a$, cannot have a stationary value strictly inside $R$ other than one for which $\boldsymbol{\alpha}, \boldsymbol{\beta}, \boldsymbol{a}$ are proportional.

The necessary conditions for a stationary value are

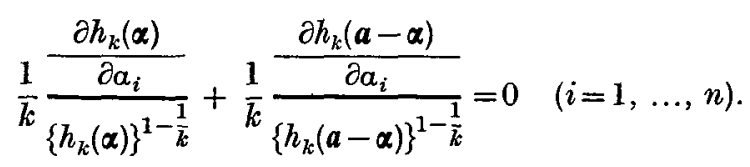

These equations are dimensionless, and so continue to hold if we replace $a-\alpha$ by $\gamma$, where $\gamma=K(\alpha-\alpha)$, and $K$ is a constant chosen so that

$$
h_{k}(\boldsymbol{\alpha})=h_{k}(\gamma)
$$

The equations then simplify to

$$
\frac{\partial h_{k}(\alpha)}{\partial \alpha_{i}}=\frac{\partial h_{k}(\gamma)}{\partial \gamma_{i}}
$$


and all that remains is to prove that the only solution to this set of equations is $\alpha=\gamma$. This will be so if the Jacobian of the $n$ functions $\partial h_{k}(\alpha) / \partial \alpha_{i}$ with respect to the $n$ variables $\alpha_{i}$ does not vanish, i.e. if the Hessian

does not vanish.

$$
\left|\frac{\partial^{2} h_{k}(\alpha)}{\partial \alpha_{r} \partial \alpha_{s}}\right|
$$

Each element in this Hessian consists of a sum of terms of the type

where

$$
A_{r, s} a_{1}^{b_{1}} a_{2}^{b_{2}} \ldots a_{n}^{b_{n}},
$$

$$
b_{1}+b_{2}+\ldots+b_{n}=k-2 \text {, }
$$

and $A_{r, 8}$ is some constant independent of $a_{1}, a_{2}, \ldots, a_{n}$. It is obvious that in fact

$$
\begin{aligned}
& A_{r, s}=\left(b_{r}+1\right)\left(b_{s}+1\right) \quad(r \neq s), \\
& A_{r, r}=\left(b_{r}+1\right)\left(b_{r}+2\right) .
\end{aligned}
$$

We now turn to a theorem due to Boole (3). [See also Muir, Theory of Determinants, Vol. III, p. 98 et seq.] This runs as follows : if an axisymmetric determinant has all its elements of the form

$$
\lambda a+\mu b+\nu c+\ldots,
$$

and if all the coefficients $\lambda, \mu, \nu, \ldots$ in all the diagonal elements are positive, and if also all the coefficients joined to any one of the variables in any one row are in order proportional to the coefficients of the same variable in any other row, then the expansion of the determinant is positively unisignant.

The Hessian with which we are dealing satisfies all these requirements except that the coefficients in the diagonal elements are, in view of (7.1), larger than they should be to give proportionality. In other words, the Hessian may be written as

$$
\left|a_{r, s}+b_{r, s}\right| \text {, }
$$

where $\left|a_{r, s}\right|$ is a determinant of the type discussed by Boole, while $\left|b_{r, s}\right|$ is a determinant which has positive elements on the principal diagonal and zeros elsewhere. If we now expand $\left|a_{r, s}+b_{r, s}\right|$ in the usual way as a sum of determinants, it is at once obvious that the expansion is positively unisignant, which completes the proof of the inequality.

\section{REFERENCES}

(1) M. Marcus and L. Lopes, Canadian J. of Math., 9 (1957), 305-12.

(2) J. J. Sylvester, Quart. J. of Math., 1 (1855), 42-56.

(3) G. Boole, Phil. Trans. Roy. Soc., 152 (1 862), 225-52.

The Mathematical Institute

16 Chambers Street

Edinburah, 1 